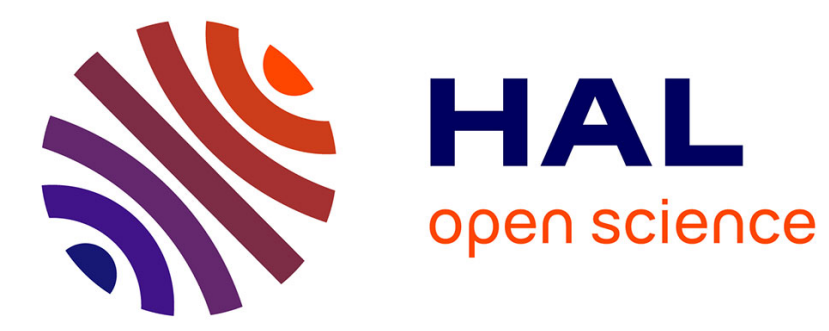

\title{
Symmetry-breaking dynamics of the modulational instability spectrum
}

M. Droques, B. Barviau, Alexandre Kudlinski, M. Taki, A. Boucon, T. Sylvestre, Arnaud Mussot

\section{- To cite this version:}

M. Droques, B. Barviau, Alexandre Kudlinski, M. Taki, A. Boucon, et al.. Symmetry-breaking dynamics of the modulational instability spectrum. Optics Letters, 2011, 36 (8), pp.1359-1361. 10.1364/OL.36.001359 . hal-00655058

\section{HAL Id: hal-00655058 \\ https://hal.science/hal-00655058}

Submitted on 19 Apr 2021

HAL is a multi-disciplinary open access archive for the deposit and dissemination of scientific research documents, whether they are published or not. The documents may come from teaching and research institutions in France or abroad, or from public or private research centers.
L'archive ouverte pluridisciplinaire HAL, est destinée au dépôt et à la diffusion de documents scientifiques de niveau recherche, publiés ou non, émanant des établissements d'enseignement et de recherche français ou étrangers, des laboratoires publics ou privés. 


\title{
Symmetry-breaking dynamics of the modulational instability spectrum
}

\author{
M. Droques, ${ }^{1}$ B. Barviau, ${ }^{1}$ A. Kudlinski, ${ }^{1}$ M. Taki, ${ }^{1}$ A. Boucon, ${ }^{2}$ T. Sylvestre, ${ }^{2}$ and A. Mussot ${ }^{1, *}$ \\ ${ }^{1}$ Université Lille 1, Laboratoire PhLAM, UMR CNRS 8523, IRCICA, 59655 Villeneuve d'Ascq Cedex, France \\ ${ }^{2}$ Institut FEMTO-ST, Département d'optique, Université de Franche-Comté, UMR CNRS 6174, 25030 Besançon, France \\ *Corresponding author: mussot@phlam.univ-lille1.fr
}

Received January 21, 2011; revised March 11, 2011; accepted March 11, 2011;

posted March 16, 2011 (Doc. ID 141565); published April 8, 2011

\begin{abstract}
We demonstrate in an optical fiber that third-order dispersion yields an unexpected symmetry-breaking dynamics of the modulational instability spectrum. It is found in particular that this spectral asymmetry does not smoothly and monotonically increase when approaching the zero-dispersion wavelength. Instead, it exhibits several local extrema and it can even be reversed at a particular dispersion value. We interpret this behavior as resulting from interactions between dispersive waves and solitons generated from modulation instability.
\end{abstract}

Modulational instability (MI) is a fundamental and ubiquitous phenomenon whereby weak modulations of an optical beam are amplified when nonlinear effects are perfectly balanced by linear ones. This leads to the breakup of the optical field into pulses or filaments and to the generation of two symmetrical spectral sidebands. In fiber optics, MI was recognized early as a simple way of generating optical solitons trains [1], and it has later been used for optical parametric amplification [2]. Analytically, the overall MI or parametric gain can be derived from a linear stability analysis of the nonlinear Schrödinger equation (NLSE) [3]. This analysis assumes two symmetric side lobes and leads to a phase-matching condition that only depends on even-order dispersion terms. The limit of this analysis has been pointed out recently by demonstrating that, in very low dispersion regions, the third-order dispersion term $\left(\beta_{3}\right)$ must be accounted for in correctly modelling the dynamics of the system [46]. This leads to a symmetry breaking of the MI spectrum and to the generation of dispersive waves (DWs) on the anti-Stokes side of the spectrum [4]. Note that these waves that originate from perturbations of solitons $[7,8]$ have been extensively studied in the context of supercontinuum generation [9].

In this Letter, we demonstrate both numerically and experimentally that third-order dispersion yields an unexpected symmetry-breaking dynamics of the amplitude of the MI spectral sidebands when they overlap with DWs. We show in particular that the induced spectral asymmetry does not monotonically increase with the second-order dispersion term $\left(\beta_{2}\right)$, but rather exhibits local extrema due to interactions between DWs and solitons. We further show that the asymmetry can even be reversed when DWs fall on the MI anti-Stokes band.

The generalized NLSE has been numerically integrated for a $6 \mathrm{~km}$-long dispersion-shifted fiber (DSF) by using an adaptive step-size method [10]. A continuous wave (CW) monochromatic pump with quantum noise corresponding to a half-photon per spectral mode has been used as the input condition in the anomalous dispersion region with the set of parameters listed in Fig. 1's caption. The stimulated Raman scattering (SRS) has been neglected in the simulations because the CW power $\left(P_{P}=790 \mathrm{~mW}\right)$ is far below the calculated Raman threshold (about $5 \mathrm{~W}$ )
[3]. Random initial conditions lead to noisy output spectra while experimental ones are smoothed due to the averaging performed during the recording. In order to be as close as possible to experimental conditions, we computed MI spectra over 50 runs for each $\beta_{2}$ value, which corresponds to a good tradeoff between time consumption and accuracy. Figure 1(a) shows the results of MI spectra as a function of $\beta_{2}$. To get a better insight, we have also plotted in Figs. 1(b)-1(g) a set of six MI spectra denoted by arrows in Fig. 1(a). Figure 1(g) illustrates the usual situation in which two nearly symmetric spectral

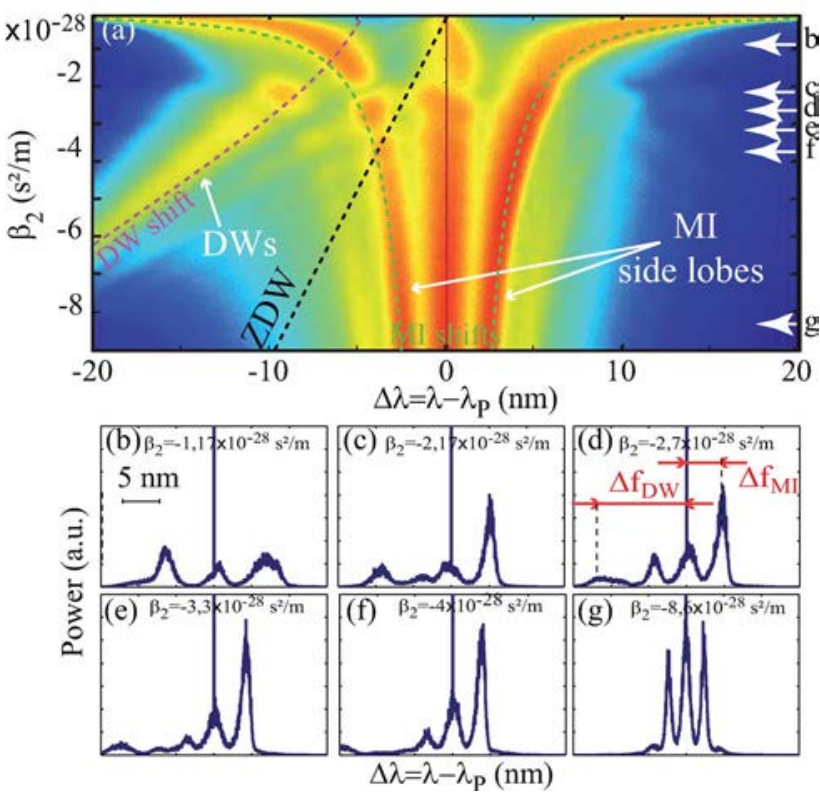

Fig. 1. (Color online) Numerical simulations. (a) Top view of MI spectrum evolution versus second-order dispersion, $\beta_{2}$, in logarithmic scale. Dashed black line, ZDW position; dashed pink curve, DW position; dashed green curves, MI sidebands calculated from standard theory [3]. (b) to (g) Spectra corresponding to $\beta_{2}$ values pointed out by arrows along right side of (a), in linear scale. Simulations parameters are $L=$ $6000 \mathrm{~m}, \quad \gamma=2.4 \mathrm{~W}^{-1} \cdot \mathrm{km}^{-1}, \quad \beta_{3}=1.2 \times 10^{-40} \mathrm{~s}^{3} / \mathrm{m}, \quad \alpha=$ $0.2 \mathrm{~dB} / \mathrm{km}$, and $P_{P}=790 \mathrm{~mW}$. A movie (Media 1) representing the spectrum evolution with $\beta_{3}=0$ (red line) and $\beta_{3}=$ $1.2 \times 10^{-40} \mathrm{~s}^{3} / \mathrm{m}$ (blue curves) in logarithmic scale can be seen online. 
sidebands appear around the pump wavelength for large $\left|\beta_{2}\right|$ values. Decreasing the $\left|\beta_{2}\right|$ value allows the MI sidebands to move away from the pump, as expected from the standard theory [3], depicted as a green dashed line in Fig. 1(a). This figure also shows a wavepacket appearing at the anti-Stokes side of the MI spectrum, which moves near the MI sidebands when $\left|\beta_{2}\right|$ decreases. This anti-Stokes spectral feature is the signature of DWs shed by solitons [8] as confirmed by the pink dashed curve in Fig. 1(a) that shows their spectral position from the theoretical phase-matching relation $\left\{\beta_{3}\left(2 \pi \Delta f_{\text {DW }}\right)^{3}+\right.$ $3 \beta_{2}\left(2 \pi \Delta f_{\mathrm{DW}}\right)^{2}-3 \gamma P_{P}=0$ [3]\}. In addition to DW generation, the Stokes-MI sideband exhibits a strong power increase due to the so-called spectral soliton recoil effect [8] for momentum conservation. This situation is clearly illustrated by the spectra of Figs. 1(c)-1(f). As long as their spectral position is far from the MI sidebands, or equivalently $\Delta f_{\mathrm{DW}}>\Delta f_{\mathrm{MI}}$, they do not strongly interact [4]. By further decreasing the $\left|\beta_{2}\right|$ value, DWs and MI side lobes spectrally overlap, which leads to an important and unexpected modification of the spectrum. For the lowest $\left|\beta_{2}\right|$ values, the asymmetry is reversed and the antiStokes MI side lobe is now stronger than the Stokes one [Fig. 1(b)]. But the most striking feature can be seen from Fig. 1(c), where the anti-Stokes MI sideband almost disappears. This situation is clearly illustrated as a spectral hole in Fig. 1(a) and appears when the modulation frequency is very close to the DW one.

Corresponding experiments have been performed in the same conditions as in simulations to get a fair comparison. We used a $6 \mathrm{~km}$-long dispersion-shifted fiber with a zero-dispersion wavelength (ZDW) located at $1550.7 \mathrm{~nm}$ and a linearly polarized tunable $\mathrm{CW}$ laser. This laser was phase-modulated to mitigate stimulated Brillouin scattering with the same technique as in [11]. The laser wavelength was tuned over $10 \mathrm{~nm}$ from the ZDW, which corresponds to a variation of the $\beta_{2}$ parameter of about $10 \times 10^{-28} \mathrm{~s}^{2} \mathrm{~m}^{-1}$. The experimental parameters are the same as those listed in Fig. 1's caption, except the pump power, which was adjusted to $630 \mathrm{~mW}$ in experiments. It corresponds to the optimal value that allows the clearest illustration of the symmetry-breaking dynamics of the MI spectrum, as discussed hereafter. Experimental MI spectra were recorded with an optical spectrum analyzer and are depicted in Fig. 2 with the same representation as in Fig. 1. As can be seen, the agreement between numerical simulations (Fig. 1) and experiments (Fig. 2) is excellent. Specifically, Figs. 1(a) and 2(a) look very similar, and interactions between DWs and the anti-Stokes MI sideband are also clearly evidenced in the experiment. Spectra displayed in Figs. 2(b)-2(g) also have the same shapes as the simulated ones of Figs. 1(b)-1(g). We also clearly observe the reversal of the symmetry breaking in Fig. 2(b) as well as an almost suppression of the anti-Stokes MI side lobe in Fig. 2(c). To go into further detail, we show in Fig. 3 the amplitude ratio [labeled $R_{P}$; see in Fig. 2(d), for example] between the maximum power of the Stokes and anti-Stokes MI side lobes as a function of $\beta_{2}$. The evolution of the $R_{P}$ ratio for increasing $\beta_{2}$ is rather unexpected from the basic MI theory, and exhibits in particular three local maxima and minima. Dashed lines in Fig. 3 were obtained (i) from numerical simulations presented
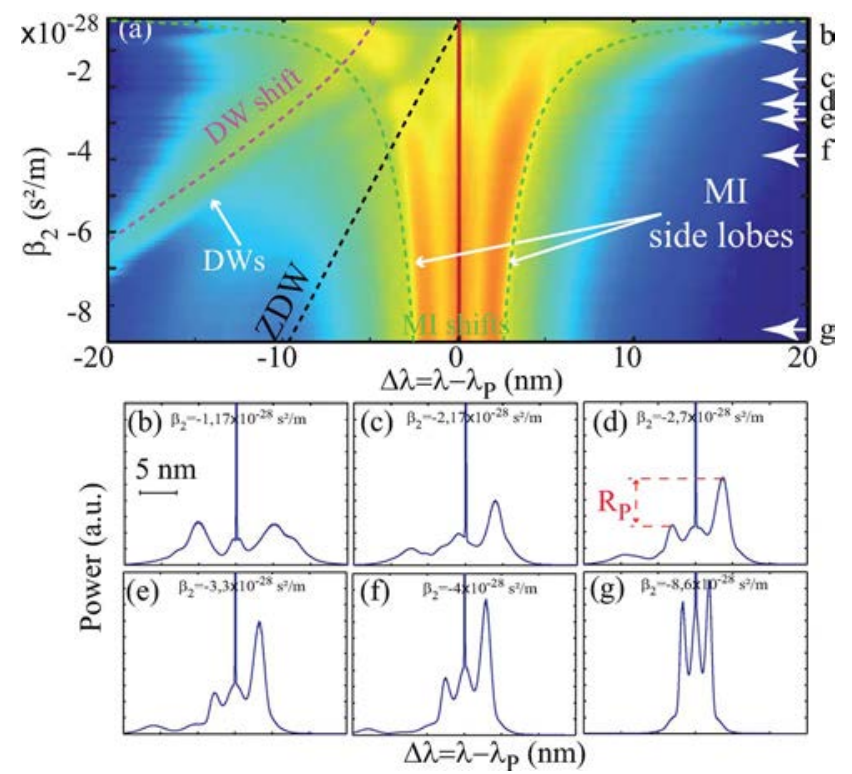

Fig. 2. (Color online) Experimental results. Same representation as in Fig. 1, and same parameters except pump power (630 $\mathrm{mW}$ compared to $790 \mathrm{~mW}$ in simulations).

in Fig. 1 ( $790 \mathrm{~mW}$ pump power), and (ii) from numerical simulations realized at different pump powers $(700 \mathrm{~mW}$ in dashed-dotted lines and $740 \mathrm{~mW}$ in dotted line). Experimental results are depicted as a solid line for a $630 \mathrm{~mW}$ pump power. The dynamics of the system can be divided into three areas. The first one (depicted in green) corresponds to the strongest $\left|\beta_{2}\right|$ values when DWs are generated far away from the MI side lobes. The second one (depicted in red) corresponds to the lowest $\left|\beta_{2}\right|$ values when DWs are generated on the anti-Stokes MI side lobe $\left(R_{P} \leq 1\right)$, and the third one sits between the first two (see Fig. 3). In the first region (in green), $\beta_{3}$ plays a minor role because solitons are relatively long temporally and the dispersion variation over

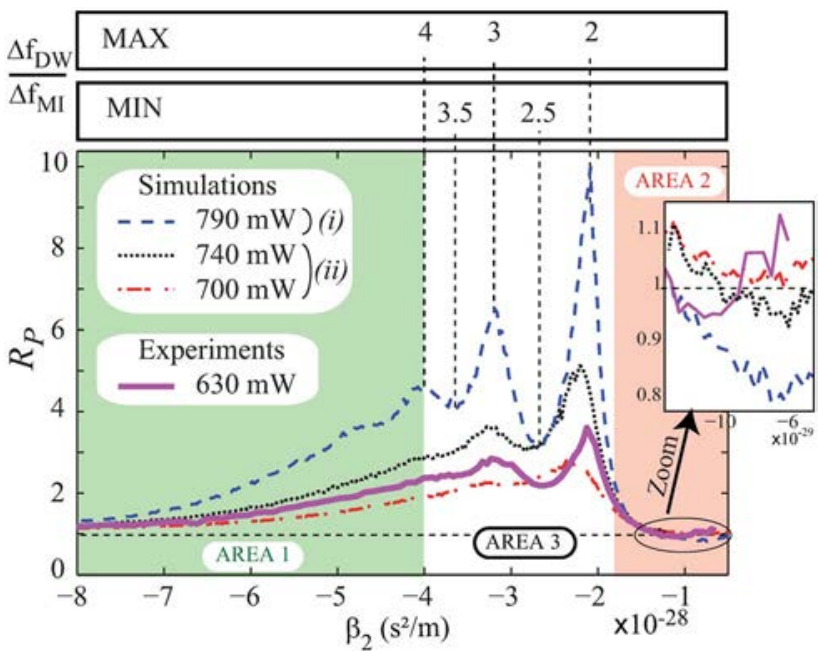

Fig. 3. (Color online) Evolution of the $R_{P}$ ratio between the Stokes and anti-Stokes MI side lobes power as a function of $\beta_{2}$. Solid curve, experimental results for a pump power of $630 \mathrm{~mW}$; dashed and/or dotted curves, numerical simulations for different pump powers. Top rectangles, corresponding ratio between DWs $\left(\Delta f_{\mathrm{DW}}\right)$ and solitons $\left(\Delta f_{\mathrm{MI}}\right)$ spectral detuning from pump. 
their spectrum is very small. As a consequence, the solitonic train is slightly disturbed, the asymmetry in power is thus very low $\left(R_{P} \rightarrow 1\right)$ and output spectra look like usual MI spectra $\left(\beta_{3}=0\right)$. By decreasing the $\left|\beta_{2}\right|$ value, solitons become shortened temporally so that the $\beta_{2}$ variation over their spectrum is now significant and leads to the generation of powerful DWs. This is associated with an increase of the $R_{P}$ ratio. In the second region (in red), DWs are generated on the anti-Stokes MI side lobe, which leads to a reversal of the asymmetry $\left(R_{P} \leq 1\right)$. The most interesting region is the last one (in white), where $R_{P}$ presents local extrema, which was a priori unexpected. DWs are indeed located relatively far from the MI side lobes, and direct interactions between these waves cannot be invoked to explain this behavior. We then look for possible interplay between the DWs and the harmonics of the MI side lobes. We calculated the value of the ratio between the spectral position of the solitons [labeled $\Delta f_{\mathrm{MI}}$ in Fig. $1(\mathrm{~d})$ ] and that of the DWs [labeled $\Delta f_{\mathrm{DW}}$ in Fig. 1(d)]. We found that all the maxima of $R_{P}$ in Fig. 3 correspond to exact integer values of this ratio, while minima are equal to exact half-integer ones (see for instance an illustration with the dashed curve corresponding to $P_{p}=790 \mathrm{~mW}$ in Fig. 3). This means that when DWs are generated exactly on one harmonic of the MI sideband, the spectral asymmetry between Stokes and antiStokes side lobes is maximum. On the contrary, when DWs are generated exactly between MI harmonics, this spectral asymmetry is minimum. This is illustrated in Fig. 3 with the $790 \mathrm{~mW}$ pump power case (dashed lines), but has been verified for all other pump power values $(700 \mathrm{~mW}$ and $740 \mathrm{~mW}$ ) investigated in numerical simulations, as well as for the experimental results represented by the solid line. When comparing the experimental curve (solid lines) with the ones obtained from simulations, the dynamics of the MI spectrum symmetry breaking is very clear and in very good agreement. In experiments, $R_{P}$ is above 1 in the first area, slightly below 1 in the second one, and presents extrema in the third one, in good agreement with simulations obtained for $740 \mathrm{~mW}$, even though the pump powers are not identical. We would like to stress here that this is really satisfactory due to the extreme sensitivity of this phenomenon to the pump power. Indeed, by only increasing it from $700 \mathrm{~mW}$ to $790 \mathrm{~mW}$ (about 12\%), $R_{P}$ experiences a threefold increase (dashed-dotted and dashed lines, respectively), while we evaluated experimental uncertainties of the $\gamma P_{P}$ product value to be $20 \%$ (about $10 \%$ for each parameter).
Note that the fiber length also impacts the symmetrybreaking dynamics of the MI spectrum. Here it was chosen to get a high value of the power asymmetry $R_{P}$ curve, but generally this behavior is clearly visible as soon as a train of solitons is generated by $\mathrm{MI}(\simeq 4500 \mathrm{~m})$ and until the spectrum become strongly saturated $(\simeq 8000 \mathrm{~m})$, which leads to a merging of all spectral components [6].

In summary, we have demonstrated in an optical fiber both numerically and experimentally that third-order dispersion induces a complex symmetry-breaking dynamics of the MI spectrum. We have shown in particular that the induced spectral asymmetry exhibits strong oscillations and that it can even be reversed at particular dispersion values. Numerical simulations and experimental results were found to be in excellent agreement, and have shown that the symmetry-breaking dynamics relies on an interaction between DWs and solitons originating from the MI process. This work should contribute to a further understanding of the key phenomena at the origin of optical rogue wave formation or supercontinuum generation in the $\mathrm{CW}$ pumping regime.

This work was partly supported by the French Ministry of Higher Education and Research, the Nord-Pas de Calais Regional Council and FEDER through the "Contrat de Projets Etat Région (CPER) 2007-2013" and the "Campus Intelligence Ambiante" (CIA).

\section{References}

1. A. Hasegawa, Opt. Lett. 9, 288 (1984).

2. M. E. Marhic, Fiber Optical Parametric Amplifiers, Oscillators and Related Devices (Cambridge University, 2008).

3. G. P. Agrawal, Nonlinear Fiber Optics, 4th ed. (Academic, 2007).

4. A. Mussot, E. Lantz, H. Maillotte, T. Sylvestre, C. Finot, and S. Pitois, Opt. Express 12, 2838 (2004).

5. A. Mussot, E. Louvergneaux, N. Akhmediev, F. Reynaud, L. Delage, and M. Taki, Phys. Rev. Lett. 101, 113904 (2008).

6. C. Michel, P. Suret, S. Randoux, H. R. Jauslin, and A. Picozzi, Opt. Lett. 35, 2367 (2010).

7. P. K. A. Wai, C. R. Menyuk, Y. C. Lee, and H. H. Chen, Opt. Lett. 11, 464 (1986).

8. N. Akhmediev and M. Karlsson, Phys. Rev. A 51, 2602 (1995).

9. J. M. Dudley and J. R. Taylor, Supercontinuum Generation in Optical Fibers (Cambridge University, 2010).

10. O. V. Sinkin, R. Holzlhner, J. Zweck, and C. R. Menyuk, J. Lightwave Technol. 21, 61 (2003).

11. A. Mussot, M. L. Parquier, and P. Szriftgiser, Opt. Comm. 283, 2607 (2010). 